\title{
Lactate Dehydrogenase A as a Potential New Biomarker for Thyroid Cancer
}

Eun Jeong Ban ${ }^{1,2, *}$, Daham Kim ${ }^{3, *}$, Jin Kyong Kim ${ }^{4}$, Sang-Wook Kang ${ }^{4}$, Jandee Lee ${ }^{4}$, Jong Ju Jeong ${ }^{4}$, Kee-Hyun Nam Woong Youn Chung ${ }^{4}$, Kunhong $\mathrm{Kim}^{5}$

${ }^{1}$ Department of Surgery, Dongguk University Ilsan Hospital, Dongguk University College of Medicine, Goyang; ${ }^{2}$ Department of Surgery, Graduate School, Yonsei University College of Medicine; ${ }^{3}$ Department of Internal Medicine, Institute of Endocrine Research, Yonsei University College of Medicine; Departments of ${ }^{4}$ Surgery, ${ }^{5}$ Biochemistry and Molecular Biology, Yonsei University College of Medicine, Seoul, Korea

Background: Several cancers show increased levels of lactate dehydrogenase A (LDHA), which are associated with cancer progression. However, it remains unclear whether LDHA levels are associated with papillary thyroid cancer (PTC) aggressiveness or with the presence of the PTC prognostic marker, the $\mathrm{BRAF}^{\mathrm{V} 600 \mathrm{E}}$ mutation. This study aimed to evaluate the potential of LDHA as a PTC prognostic marker.

Methods: LDHA expression was examined in 83 PTC tissue specimens by immunohistochemistry. Human thyroid cell lines were genetically manipulated to overexpress $\mathrm{BRAF}^{\mathrm{V} 600 \mathrm{E}}$ or were treated with a BRAF-specific short hairpin RNA (shBRAF), whose effects on LDHA expression were evaluated by Western blotting. Data from 465 PTC patients were obtained from The Cancer Genome Atlas (TCGA) database and analyzed to validate the in vitro results.

Results: LDHA was aberrantly overexpressed in PTC. Intense immunostaining for LDHA was observed in PTC specimens carrying mutated BRAF, whereas the intensity was less in wild-type BRAF samples. Overexpression of BRAF ${ }^{\mathrm{V} 600 \mathrm{E}}$ resulted in LDHA upregulation, whereas treatment with shBRAF downregulated LDHA in human thyroid cell lines. Furthermore, $L D H A$ mRNA expression was significantly elevated and associated with $\mathrm{BRAF}^{\mathrm{V} 600 \mathrm{E}}$ expression in thyroid cancer tissues from TCGA database. Additionally, LDHA overexpression was found to be correlated with aggressive clinical features of PTC, such as lymph node metastases and advanced tumor stages.

Conclusion: $L D H A$ overexpression is associated with the $\mathrm{BRAF}^{\mathrm{V} 600 \mathrm{E}}$ mutation and an aggressive PTC behavior. Therefore, LDHA may serve as a biomarker and therapeutic target in PTC.

Keywords: Biomarkers; BRAF; Lactate dehydrogenase A; Thyroid cancer, papillary; Prognosis

\section{INTRODUCTION}

Cancer metabolism, which exploits dysregulated metabolic pathways, is characterized by increased glycolysis [1]. In normal cells, adenosine triphosphate is mostly produced by mitochondrial oxidative phosphorylation, while cancer cells tend to
Received: 18 August 2020, Revised: 4 December 2020,

Accepted: 31 December 2020

Corresponding author: Kee-Hyun Nam

Department of Surgery, Yonsei University College of Medicine, 50-1 Yonsei-ro, Seodaemun-gu, Seoul 03722, Korea

Tel: +82-2-2228-2100, Fax: +82-2-313-8289, E-mail: khnam@yuhs.ac

\section{Copyright (C) 2021 Korean Endocrine Society}

This is an Open Access article distributed under the terms of the Creative Commons Attribution Non-Commercial License (https://creativecommons.org/ licenses/by-nc/4.0/) which permits unrestricted non-commercial use, distribution, and reproduction in any medium, provided the original work is properly cited.

\footnotetext{
*These authors contributed equally to this work.
} 
use glycolysis, despite the presence of oxygen. Named Warburg effect, this process relies on lactate dehydrogenase A (LDHA) to convert pyruvate to lactate in the final step of glycolysis [2]. LDHA is the key enzyme in glycolysis.

Several studies have revealed high expression of LDHA in various cancers and its association with cancer progression. In esophageal squamous cell carcinoma, LDHA expression is upregulated, whereas LDHA knockdown inhibits cell growth and migration in vitro and impairs tumorigenesis in vivo [3]. LDHA expression is also upregulated in primary clear cell renal carcinoma, wherein LDHA shows potential utility as a prognostic marker [4]. Significantly elevated LDHA has also been observed in patients with gastric cancer, and LDHA expression was reported to be correlated with lower overall survival in these patients [5].

Thyroid cancer is the most common endocrine malignancy [6]. The rising incidence of thyroid cancer, especially papillary thyroid cancer (PTC), is a worldwide phenomenon $[7,8]$. The $\mathrm{BRAF}^{\mathrm{V} 600 \mathrm{E}}$ mutation, which is the most common driver mutation in PTC, is known to have aggressive features, such as extrathyroidal extension, lymph node metastasis, and an advanced cancer stage [9-11]. However, the BRAF ${ }^{\mathrm{V} 600 \mathrm{E}}$ mutation is not an independent predictor of PTC, and novel prognostic markers are needed. Recently, some progress has been achieved regarding thyroid tumor metabolism [12]. Previous studies have demonstrated that the expression of glycolytic enzymes is upregulated in thyroid cancer $[13,14]$. However, it is not entirely clear whether LDHA levels are associated with the aggressiveness of PTC or the presence of BRAF ${ }^{\mathrm{V} 600 \mathrm{E}}$ mutation [15]. Considering the pivotal role of LDHA in the regulation of metabolic pathways, its implication in the metabolic switch of PTC warrants further investigation.

This study aimed to evaluate the potential of LDHA as a prognostic marker of clinicopathological features and clinical outcomes in PTC.

\section{METHODS}

\section{Human tissue samples}

Human thyroid cancer tissue samples $(n=83)$ and matched contralateral normal tissue samples $(n=81)$, who underwent thyroidectomy for PTC between October 2005 and April 2014 at the Yonsei University Severance Hospital (Seoul, Korea), were obtained. We included patients whose preoperative 2-deoxy$2-\left[{ }^{18} \mathrm{~F}\right]$ fluoro-D-glucose (FDG) positron emission tomography integrated with computed tomography imaging and $\mathrm{BRAF}^{\mathrm{V} 600 \mathrm{E}}$ mutation results were available. Patients who had a history of other cancers or autoimmune thyroiditis (proven by the presence of elevated levels of thyroid antibodies and by thyroid imaging) were excluded. All samples were obtained as formalinfixed paraffin-embedded tissue blocks. This study was conducted in accordance with the Declaration of Helsinki and was approved by the Institutional Review Board of the Severance Hospital (No. 4-2015-0677). Written informed consent was obtained from all patients included in the study.

\section{Data from The Cancer Genome Atlas thyroid cancer database}

Publicly available mRNA sequencing (RNA-Seq) data, somatic mutation data, and clinical information of 465 patients with thyroid cancer from The Cancer Genome Atlas (TCGA) database (version 2016_01_28; https://gdac.broadinstitute.org) were analyzed $[16,17]$. The overall survival, disease-free survival, disease-specific survival, and progression-free survival data were downloaded from cBioPortal (TCGA, Firehose Legacy, and PanCancer Atlas). All the collected data were fully anonymized before we accessed the patient information. Patients with a history of any other malignancy and those without BRAF or LDHA information were not included in the study. Thyroid cancer staging was based on the 7th edition of the American Joint Committee on Cancer (AJCC) cancer staging system.

\section{Cell culture}

Three human thyroid cell lines (Nthy-ori 3-1, SNU-790, and 8505C) were used for in vitro experiments. Nthy-ori 3-1 cells were provided by Dr. Seongho Ryu (Soonchunhyang University, Cheonan, Korea), and 8505C cells were provided by Dr. Seong Jin Lee (Hallym University, Chuncheon, Korea). SNU790 cells were purchased from the Korean Cell Line Bank (KCLB No. 00790.1, Seoul, Korea). All the cell lines were authenticated by short tandem repeat profiling and confirmed to be free of Mycoplasma contamination. Cells were cultured in RPMI-1640 medium (Gibco, Thermo Fisher Scientific, Waltham, MA, USA), supplemented with $10 \%$ fetal bovine serum (HyClone, Logan, UT, USA) and 1\% penicillin/streptomycin, in a humidified atmosphere of $5 \% \mathrm{CO}_{2}$ and $95 \%$ air at $37^{\circ} \mathrm{C}$.

\section{Transfection}

pCDH-B-Raf and pCDH-B-RafV600E vectors were kindly provided by Tae Jun Park [18]. Lentiviral particles were generated using three plasmids, VSVG, RSV-REV, and PMDLg/ 
pPRE, cotransfected with the pCDH-B-Raf or pCDH-BRafV600E plasmid in HEK293FT cells (R70007, Invitrogen, Carlsbad, CA, USA) using the Lipofectamine 2000 transfection reagent (\#11668019, Invitrogen). Two days after the transfection, the culture medium was collected and passed through a $0.45-\mu \mathrm{m}$ filter. The purified lentiviral particles were used to infect Nthy-ori 3-1 cells.

The BRAF-specific short hairpin RNA (shRNA) and a scrambled negative control were purchased from OriGene (Rockville, MD, USA). Cells were seeded onto 6-well plates and transfected with the shRNA using Lipofectamine 2000 (Invitrogen). The shRNA-transfected cells were subjected to Western blot analysis 72 hours after transfection.

\section{Western blot analysis}

Western blot analysis was performed as previously described in a study [19]. Membranes were immunoassayed with antibodies specific to the following antigens: LDHA $(1: 1,000 ; 3582 S)$, extracellular signal-regulated kinase 1/2 (ERK1/2; 1:1,000; 9102S), phosphorylated ERK1/2 (pERK1/2; 1:1,000; 9106S, Cell Signaling Technology, Danvers, MA, USA), $\beta$-actin (1:5,000; A1978, Sigma-Aldrich, St. Louis, MO, USA), and BRAF (1:1,000; sc-5284, Santa Cruz Biotechnology, Dallas, TX, USA). The secondary antibodies included horseradish peroxidase-conjugated goat anti-rabbit immunoglobulin G (IgG; $1: 5,000 ; 31460)$ and goat anti-mouse $\operatorname{IgG}(1: 5,000 ; 31430$, Thermo Fisher Scientific). Signals were visualized using Amersham ECL Western blotting detection reagents (GE Healthcare Life Sciences, Chicago, IL, USA).

\section{Immunohistochemistry}

Paraffin-embedded tissue specimens were cut into 4- $\mu$ m-thick sections, and immunohistochemistry (IHC) staining was performed using a Discovery XT autoimmunostainer (750-701, Ventana, Tucson, AZ, USA) with monoclonal antibodies against glucose transporter 1 (GLUT1; 1:100; sc-377228, Santa Cruz Biotechnology), pyruvate kinase isoform M2 (PKM2; 1:500; 4053S), and LDHA (1:100; 3582S, Cell Signaling Technology), according to the manufacturer's instructions.

The IHC results were assessed by two experts using immunoreactive scoring by multiplying the intensity of staining (I) (0-3 points: absent, weak, intermediate, and strong, respectively) with the percentage of positive tumor cells $(\mathrm{P})(0-4$ points: $0 \%$, $1 \%-24 \%, 25 \%-49 \%, 50 \%-74 \%$, and $75 \%-100 \%$, respectively). The final calculated immunoreactive score $(\mathrm{H}=\mathrm{P} \times \mathrm{I})$ was evaluated according to the following scale: low (0-4 points), moderate (6-8 points), and high (9-12 points).

\section{Statistical analysis}

IBM SPSS Statistics version 25 (IBM, Armonk, NY, USA) was used for all statistical analyses. Categorical variables are presented as the number and percentage, and continuous variables are presented as the mean \pm standard deviation. Statistical comparisons of mean values were performed using Student's $t$ test for two groups and analysis of variance for more than two groups. As an exception, the Mann-Whitney $U$ test and KruskalWallis test were used to compare the mRNA expression results, as they usually do not present a normal distribution. Group comparisons were performed using the chi-square test or a linear-by-linear association test, as appropriate. Survival analyses were performed using Gehan's generalized Wilcoxon method. Univariate and multivariate analyses were used to determine the variables independently associated with lymph node metastasis. Odds ratios and $95 \%$ confidence intervals were determined. Differences with a $P$ value $<0.05$ were considered statistically significant.

\section{RESULTS}

\section{Expression of glycolytic enzymes in human thyroid cancer tissues}

To evaluate whether glycolysis is increased in PTC, the levels of important glycolytic enzymes, such as GLUT1, PKM2, and LDHA were compared among 83 cancer specimens and 81 matched normal specimens from the same patients by immunohistochemical staining (Fig. 1). Basal characteristics of 83 patients included in the study are shown in Supplemental Table S1. GLUT1, PKM2, and LDHA levels were significantly upregulated in the thyroid cancer tissues compared with their normal counterparts $(P<0.001)$ (Table 1$)$.

Next, glycolytic enzyme levels were compared according to the BRAF ${ }^{\mathrm{V} 600 \mathrm{E}}$ mutational status. Of the 83 patients with thyroid cancer, $71(85.5 \%)$ had PTC-positive $\mathrm{BRAF}^{\mathrm{V} 600 \mathrm{E}}$ mutation, and 12 (14.5\%) had wild-type (WT) BRAF. GLUT1 and PKM2 levels were similar between the $\mathrm{BRAF}^{\mathrm{V} 600 \mathrm{E}}$ and WT BRAF groups, whereas LDHA level was significantly higher in the BRAF ${ }^{\mathrm{V} 600 \mathrm{E}}$ group ( $P=0.020$ ) (Table 2 ). These results demonstrate a clear association between the levels of LDHA and the presence of the $\mathrm{BRAF}^{\mathrm{V} 600 \mathrm{E}}$ mutation in patients with PTC.

\section{LDHA expression in human thyroid cell lines}

To investigate whether the presence of the $\mathrm{BRAF}^{\mathrm{V} 600 \mathrm{E}}$ mutation 

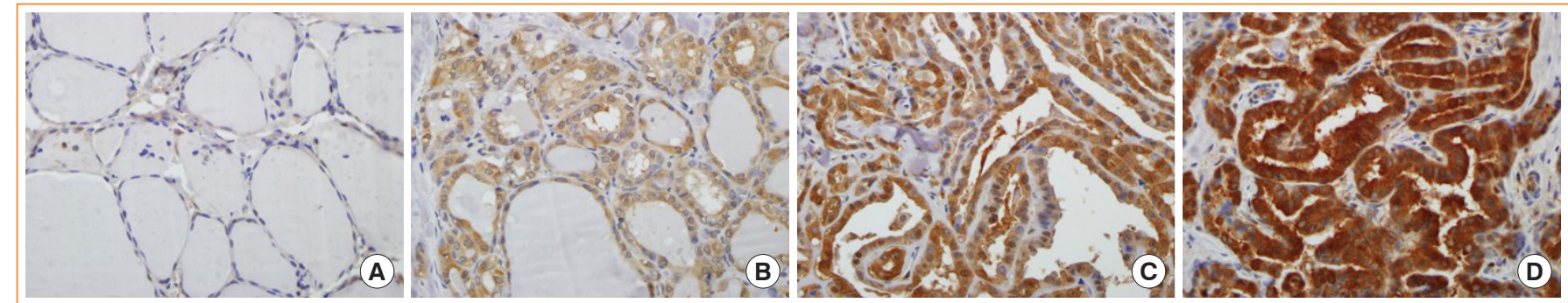

Fig. 1. Lactate dehydrogenase A (LDHA) expression in normal and tumor tissues analyzed by immunohistochemistry. (A) Normal thyroid tissue; (B) weakly, (C) intermediately, and (D) strongly stained papillary thyroid carcinoma tissues $(\times 400)$.

Table 1. Glycolytic Enzyme Level in Normal Thyroid and Papillary Thyroid Cancer Tissues

\begin{tabular}{lccc}
\hline Variable & $\begin{array}{c}\text { Normal } \\
(n=81)\end{array}$ & $\begin{array}{c}\text { Cancer } \\
(n=83)\end{array}$ & $P$ value \\
\hline GLUT1 expression & & & $<0.001$ \\
Low & $80(98.8)$ & $8(9.6)$ & \\
Moderate & $1(1.2)$ & $71(85.5)$ & \\
High & 0 & $4(4.8)$ & \\
PKM2 expression & & & $<0.001$ \\
Low & $79(97.5)$ & $2(2.4)$ & \\
Moderate & $2(2.5)$ & $22(26.5)$ & \\
High & 0 & $59(71.1)$ & \\
LDHA expression & & & $<0.001$ \\
Low & $80(98.8)$ & $6(7.2)$ & \\
Moderate & $1(1.2)$ & $21(25.3)$ & \\
High & 0 & $56(67.5)$ &
\end{tabular}

Values are expressed as number (\%).

GLUT1, glucose transporter 1; PKM2, pyruvate kinase isoform M2;

LDHA, lactate dehydrogenase A.

leads to the upregulation of LDHA expression in thyroid cancer, LDHA level was analyzed in BRAF-overexpressing thyroid cells. BRAF ${ }^{\mathrm{V} 600 \mathrm{E}}$-overexpressing Nthy-ori 3-1 cells had a spindle-transformed shape that may be the result of epithelial-mesenchymal transition induced by the BRAF ${ }^{\mathrm{V} 600 \mathrm{E}}$ mutation (Fig. 2A). BRAF ${ }^{\mathrm{V} 600 \mathrm{E}}$-overexpressing Nthy-ori 3-1 cells showed significantly higher levels of pERK than WT BRAF-overexpressing cells (Fig. 2B), as well as high levels of LDHA. Thus, overexpression of $\mathrm{BRAF}^{\mathrm{V} 600 \mathrm{E}}$ in vitro activated the mitogen-activated protein kinase signaling pathway, thereby promoting LDHA expression.

Next, the effects of BRAF inhibition on LDHA expression were investigated in the human thyroid cell lines. BRAF knockdown experiments were performed in SNU-790 and 8505c cells
Table 2. Association between Glycolytic Enzyme Level and the $\mathrm{BRAF}^{\mathrm{V} 600 \mathrm{E}}$ Mutation

\begin{tabular}{lccc}
\hline Variable & $\begin{array}{c}\mathrm{BRAF} \text { WT } \\
(n=12)\end{array}$ & $\begin{array}{c}\mathrm{BRAF}^{\mathrm{V} 600 \mathrm{E}} \\
(n=71)\end{array}$ & $P$ value \\
\hline GLUT1 expression & & & 0.788 \\
Low & $1(8.3)$ & $7(9.9)$ & \\
Moderate & $10(83.3)$ & $61(85.9)$ & \\
High & $1(8.3)$ & $3(4.2)$ & \\
PKM2 expression & & & 0.145 \\
Low & 0 & $2(2.8)$ & \\
Moderate & $6(50.0)$ & $16(22.5)$ & \\
High & $6(50.0)$ & $53(74.6)$ & \\
LDHA expression & & & \\
Low & $3(25.0)$ & $3(4.2)$ & \\
Moderate & $4(33.3)$ & $17(23.9)$ & \\
High & $5(41.7)$ & $51(71.8)$ &
\end{tabular}

Values are expressed as number (\%).

WT, wild-type; GLUT1, glucose transporter 1; PKM2, pyruvate kinase isoform M2; LDHA, lactate dehydrogenase A.

using a BRAF-specific shRNA (shBRAF). BRAF inhibition led to a reduced expression of LDHA in these cell lines (Fig. 3). These results suggest that the presence of the $\mathrm{BRAF}^{\mathrm{V} 600 \mathrm{E}}$ mutation may lead to the upregulation of LDHA expression through the BRAF/mitogen-activated protein kinase signaling pathway in thyroid cancer.

\section{TCGA thyroid cancer data according to the $L D H A$ mRNA expression status}

To confirm our findings on $L D H A$ expression patterns in PTC and evaluate whether $L D H A$ expression is associated with the aggressiveness of PTC, as well as with the presence of the $\mathrm{BRAF}^{\mathrm{V} 600 \mathrm{E}}$ mutation, data obtained from TCGA thyroid cancer database were evaluated. First, mRNA levels of GLUT1, PKM2, 

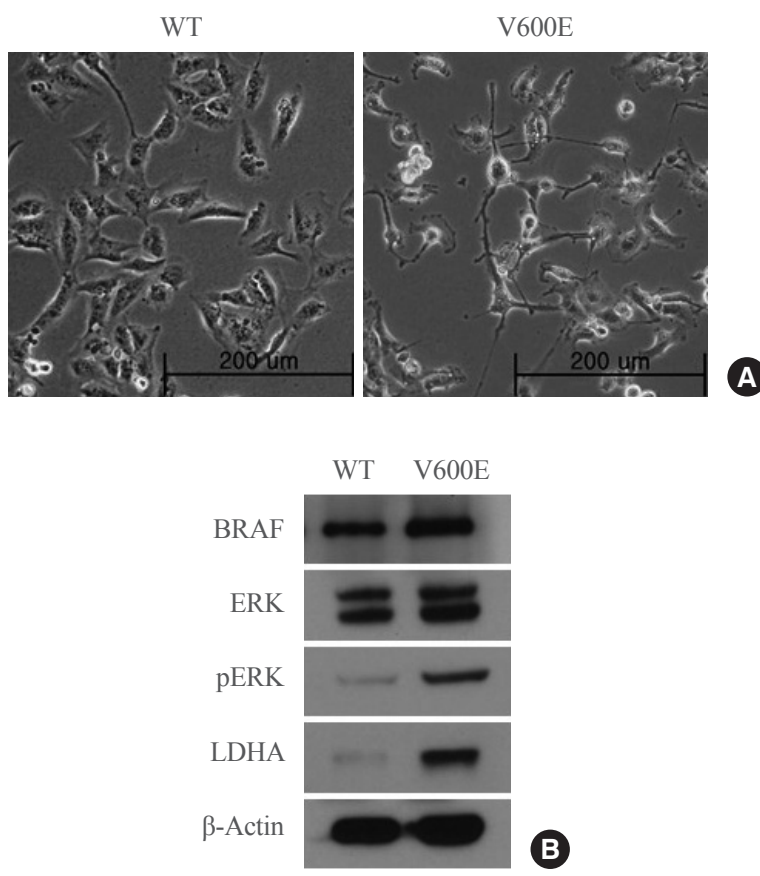

Fig. 2. Protein expression in Nthy-ori 3-1 cells stably expressing the wild-type (WT) BRAF or BRAF with the V600E mutation. (A) Photomicrographs of $\mathrm{BRAF}^{\mathrm{V} 600 \mathrm{E}}$ - and WT BRAF-overexpressing cells. (B) BRAF, extracellular signal-regulated kinase (ERK), phosphorylated ERK (pERK), lactate dehydrogenase A (LDHA), and $\beta$-actin levels in $\mathrm{BRAF}^{\mathrm{V} 600 \mathrm{E}}$ - and WT BRAF-overexpressing cells. The blot is representative of three independent experiments.
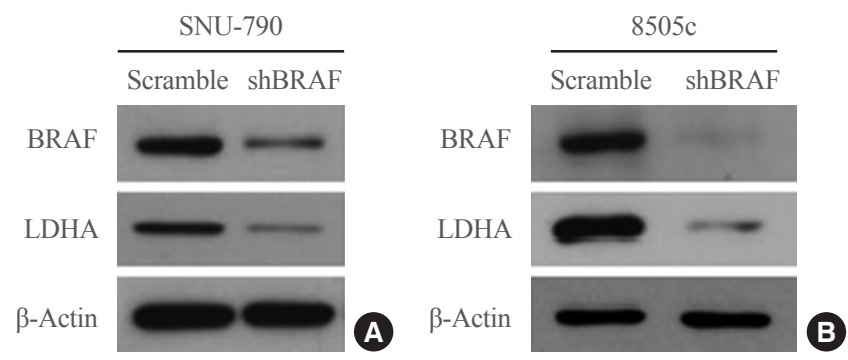

Fig. 3. Lactate dehydrogenase A (LDHA) expression in human thyroid cell lines after BRAF-specific short hairpin RNA (shBRAF) treatment. (A) SNU-790 and (B) 8505c cells. Each blot is representative of three independent experiments.

and $L D H A$ were examined, revealing that GLUT1 and PKM2 expression was significantly elevated in thyroid cancer tissues compared with normal tissues $(P<0.001)$ (Fig. 4A, B). LDHA mRNA levels were also significantly higher in thyroid cancer tissues $(P=0.016)$ (Fig. 4C). The mRNA levels of GLUT1, $P K M 2$, and $L D H A$ in paired PTC tissues and normal tissues were also examined and shown in Supplemental Fig. S1. These findings were consistent with the previous IHC results.
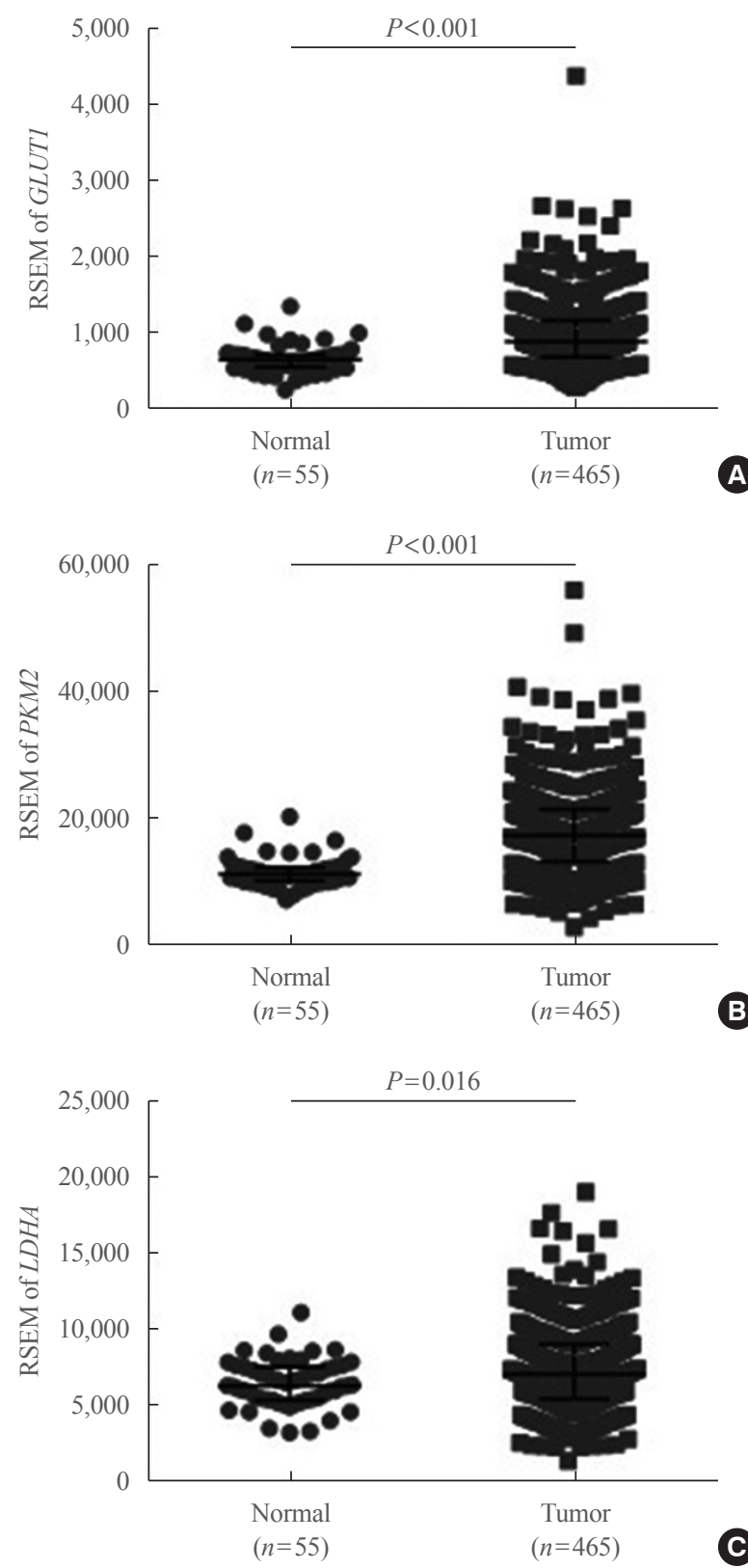

Fig. 4. mRNA expression of glycolytic enzyme-encoding genes based on The Cancer Genome Atlas thyroid cancer database. (A) Glucose transporter 1 (GLUT1), (B) pyruvate kinase isoform M2 (PKM2), and (C) lactate dehydrogenase A ( $L D H A)$ expression levels in 465 papillary thyroid carcinoma and 55 normal thyroid tissues. Data are presented as the individual value, median, and interquartile range. RSEM, RNA-Seq by expectation-maximization.

Next, the patients with PTC were divided into three groups based on the $L D H A$ expression: low $(n=155)$, moderate $(n=155)$, and high $(n=155)$ expression groups (Table 3$)$.

The analysis of the clinicopathological characteristics of the patients revealed that high $L D H A$ expression was associated 
Table 3. Clinicopathological Characteristics of Patients with Papillary Thyroid Cancer According to LDHA mRNA Expression Levels

\begin{tabular}{|c|c|c|c|c|}
\hline \multirow{2}{*}{ Characteristic } & \multicolumn{3}{|c|}{$L D H A$ expression } & \multirow{2}{*}{$P$ value } \\
\hline & Low $(n=155)$ & Moderate $(n=155)$ & $\operatorname{High}(n=155)$ & \\
\hline Age, yr & $46.85 \pm 15.73$ & $45.97 \pm 15.39$ & $46.81 \pm 15.65$ & 0.855 \\
\hline Sex & & & & 0.300 \\
\hline Male & 37 (23.9) & $38(24.5)$ & $45(29.0)$ & \\
\hline Female & $118(76.1)$ & $117(75.5)$ & $110(71.0)$ & \\
\hline Tumor size, $\mathrm{cm}$ & $2.96 \pm 1.71$ & $2.69 \pm 1.55$ & $3.06 \pm 1.61$ & 0.145 \\
\hline Extrathyroidal extension & & & & $<0.001$ \\
\hline Negative & $126(81.3)$ & $110(71.0)$ & $78(50.3)$ & \\
\hline Minimal & $25(16.1)$ & $38(24.5)$ & $58(37.4)$ & \\
\hline Moderate/advanced & $1(0.6)$ & $2(1.3)$ & $13(8.4)$ & \\
\hline Unknown & $3(1.9)$ & $5(3.2)$ & $6(3.9)$ & \\
\hline Multifocality & & & & 0.294 \\
\hline Negative & $78(50.3)$ & $86(55.5)$ & $86(55.5)$ & \\
\hline Positive & $72(46.5)$ & $67(43.2)$ & $66(42.6)$ & \\
\hline Unknown & $5(3.2)$ & $2(1.3)$ & $3(1.9)$ & \\
\hline T stage & & & & 0.001 \\
\hline $\mathrm{T} 1$ & $46(29.7)$ & $52(33.5)$ & $34(21.9)$ & \\
\hline $\mathrm{T} 2$ & $58(37.4)$ & $56(36.1)$ & $42(27.1)$ & \\
\hline $\mathrm{T} 3$ & $49(31.6)$ & $42(27.1)$ & $66(42.6)$ & \\
\hline $\mathrm{T} 4$ & $2(1.3)$ & $4(2.6)$ & $13(8.4)$ & \\
\hline $\mathrm{Tx}$ & 0 & $1(0.6)$ & 0 & \\
\hline N stage & & & & 0.039 \\
\hline N0 & $92(59.4)$ & $66(42.6)$ & $54(34.8)$ & \\
\hline N1a & $30(19.4)$ & $48(31.0)$ & $60(38.7)$ & \\
\hline N1b & $13(8.4)$ & $26(16.8)$ & $31(20.0)$ & \\
\hline $\mathrm{Nx}$ & $20(12.9)$ & $15(9.7)$ & $10(6.5)$ & \\
\hline Distant metastasis & & & & 0.050 \\
\hline M0 & $75(48.4)$ & $87(56.5)$ & $92(59.4)$ & \\
\hline M1 & $2(1.3)$ & $3(1.9)$ & $2(1.3)$ & \\
\hline $\mathrm{Mx}$ & $78(50.3)$ & $64(41.6)$ & $61(39.4)$ & \\
\hline TNM stage & & & & 0.001 \\
\hline I & $95(61.3)$ & $98(64.1)$ & $81(52.3)$ & \\
\hline II & $24(15.5)$ & $12(7.8)$ & $8(5.2)$ & \\
\hline III & $28(18.1)$ & $23(15.0)$ & $39(25.2)$ & \\
\hline IV & $8(5.2)$ & $20(13.1)$ & $27(17.4)$ & \\
\hline BRAF mutation & & & & $<0.001$ \\
\hline WT & $119(76.8)$ & $74(47.7)$ & $55(35.5)$ & \\
\hline V600E & $36(23.2)$ & $81(52.3)$ & $100(64.5)$ & \\
\hline TDS & $0.55 \pm 0.96$ & $-0.05 \pm 0.94$ & $-0.46 \pm 0.87$ & $<0.001$ \\
\hline
\end{tabular}

Values are expressed as mean \pm standard deviation or number $(\%)$.

LDHA, lactate dehydrogenase A; TNM, tumor, node, metastasis; WT, wild-type; TDS, thyroid differentiation score. 
with extrathyroidal extension, T stage, N stage, TNM stage, and $\mathrm{BRAF}^{\mathrm{V} 600 \mathrm{E}}$ mutation, but not with other parameters, such as age and sex. The thyroid differentiation score was negatively associated with high $L D H A$ levels. Moreover, univariate and multi- variate analyses confirmed that high $L D H A$ expression was associated with lymph node metastases (Table 4). These data indicate that high expression of $L D H A$ may be used as a biomarker for poor prognosis in patients with PTC. We also compared the

Table 4. Data of Univariate and Multivariate Analyses of Lymph Node Metastasis in Patients with Papillary Thyroid Cancer

\begin{tabular}{|c|c|c|c|c|c|c|}
\hline \multirow{2}{*}{ Variable } & \multicolumn{3}{|c|}{ Univariate analysis } & \multicolumn{3}{|c|}{ Multivariate analysis $^{\mathrm{a}}$} \\
\hline & OR & $95 \% \mathrm{CI}$ & $P$ value & OR & $95 \% \mathrm{CI}$ & $P$ value \\
\hline Age & 0.987 & $0.976-0.999$ & 0.038 & & & \\
\hline Female sex & 0.599 & $0.394-0.910$ & 0.016 & 0.735 & $0.461-1.174$ & 0.198 \\
\hline Multifocality & 1.461 & $1.007-2.120$ & 0.046 & 1.602 & $1.057-2.428$ & 0.026 \\
\hline \multicolumn{7}{|l|}{ Extrathyroidal extension } \\
\hline Negative & - & - & - & - & - & - \\
\hline Minimal & 2.596 & $1.688-3.992$ & $<0.001$ & 1.516 & $0.797-2.882$ & 0.205 \\
\hline Moderate/advanced & 11.948 & $2.668-53.506$ & 0.001 & 4.080 & $0.381-43.719$ & 0.245 \\
\hline Tumor size, $\mathrm{cm}$ & 1.198 & $1.062-1.351$ & 0.003 & & & \\
\hline \multicolumn{7}{|l|}{ T stage } \\
\hline $\mathrm{T} 1$ & - & - & - & - & - & - \\
\hline $\mathrm{T} 2$ & 1.600 & $0.981-2.610$ & 0.060 & 1.705 & $1.006-2.890$ & 0.048 \\
\hline $\mathrm{T} 3$ & 2.933 & $1.803-4.774$ & $<0.001$ & 2.121 & $1.074-4.188$ & 0.030 \\
\hline $\mathrm{T} 4$ & 12.267 & $3.384-44.463$ & $<0.001$ & 3.398 & $0.407-28.396$ & 0.259 \\
\hline \multicolumn{7}{|l|}{ LDHA expression } \\
\hline Low & - & - & - & - & - & - \\
\hline Moderate & 2.380 & $1.484-3.816$ & 0.001 & 2.540 & $1.507-4.283$ & $<0.001$ \\
\hline High & 3.703 & $2.303-5.957$ & $<0.001$ & 2.920 & $1.685-5.059$ & $<0.001$ \\
\hline $\mathrm{BRAF}^{\mathrm{V} 600 \mathrm{E}}$ mutation & 1.466 & $1.015-2.118$ & 0.041 & 0.962 & $0.623-1.484$ & 0.861 \\
\hline
\end{tabular}

OR, odds ratio; $\mathrm{CI}$, confidence interval; T stage, tumor stage; $L D H A$, lactate dehydrogenase $\mathrm{A}$.

${ }^{a}$ Multivariate models were adjusted for gender, multifocality, extrathyroidal extension, T stage, $L D H A$ mRNA expression, and $\mathrm{BRAF}^{\mathrm{V} 600 \mathrm{E}}$ mutation using the Enter method.
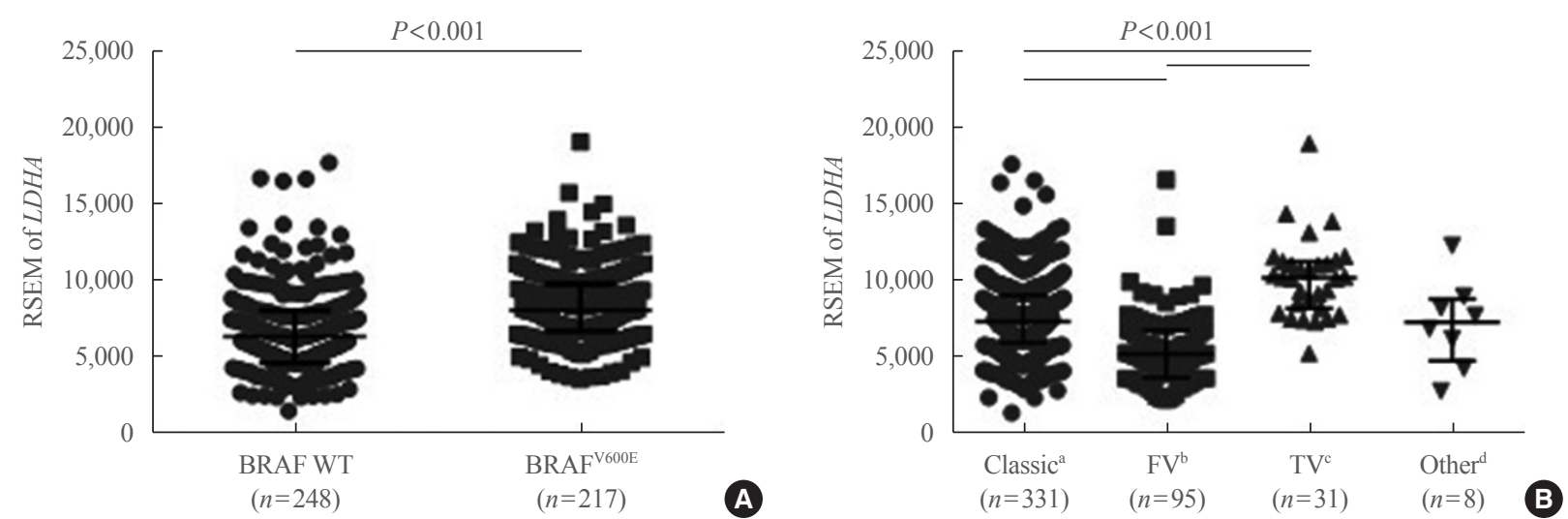

Fig. 5. Lactate dehydrogenase $\mathrm{A}(L D H A)$ gene expression levels according to the $\mathrm{BRAF}^{\mathrm{V} 600 \mathrm{E}}$ mutational status or papillary thyroid cancer (PTC) subtype, based on The Cancer Genome Atlas (TCGA) thyroid cancer database. (A) LDHA mRNA expression levels in BRAF wildtype (WT) and V600E mutant PTC. (B) LDHA mRNA expression levels according to the subtype of PTC. Data are presented as the individual value, median, and interquartile range. RSEM, RNA-Seq by expectation-maximization. ${ }^{\mathrm{a} C l a s s i c}$, classical/usual; ${ }^{\mathrm{b}} \mathrm{FV}$, follicular $(\geq 99 \%$ follicular patterned); ${ }^{\mathrm{T}} \mathrm{TV}$, tall-cell ( $\geq 50 \%$ tall cell features); ${ }^{\mathrm{d} O t h e r, ~ u n c o m m o n ~ P T C ~ v a r i a n t s . ~}$ 
survival curves of groups with different levels of LDHA expression. The overall survival, disease-free survival, disease-specific survival, and progression-free survival did not differ between the three groups $(P=0.651, P=0.244, P=0.582$, and $P=0.290$, respectively) (Supplemental Fig. S2). The mean survival rate after 10 years was higher than $90 \%$.

To further confirm the IHC findings regarding $L D H A$ expression patterns with respect to the $\mathrm{BRAF}^{\mathrm{V} 600 \mathrm{E}}$ mutational status, the latter two parameters were compared in the patient data from TCGA database. $L D H A$ expression was enhanced in patients with PTC who harbored the $\mathrm{BRAF}^{\mathrm{V} 600 \mathrm{E}}$ mutation compared with those who had the WT gene $(P<0.001)$ (Fig. 5A). Analysis of $L D H A$ expression in light of PTC subtypes, namely classical/ usual, follicular, tall-cell, and other uncommon PTC variants, revealed significantly higher LDHA level within the classical type than in the follicular variant type $(P<0.001)$ (Fig. 5B), as well as in the tall-cell variant type than in the classical type $(P<0.001)$. Taken together, these results indicate that $L D H A$ overexpression is associated with the aggressiveness of PTC, as well as with the presence of the $\mathrm{BRAF}^{\mathrm{V} 600 \mathrm{E}}$ mutation.

\section{DISCUSSION}

Although partly attributed to overdiagnosis, the incidence of thyroid cancer, particularly PTC, has increased in recent years worldwide [7,8]. Nevertheless, a decreasing trend has been reported in thyroid cancer mortality [6]. Therefore, risk stratification and tailored management, along with the identification of novel prognostic markers, are crucial, yet unmet clinical goals in thyroid cancer management [20-22]. Herein, we showed that LDHA, a key glycolytic enzyme, could be used as a potential prognostic marker in thyroid cancer.

We demonstrated that the levels of glycolytic enzymes, including LDHA, were significantly upregulated in PTC. These observations were further confirmed by the examination of the $L D H A$ expression in a set of 465 cases of PTC from TCGA thyroid cancer database. Thus, similar to previous reports [13,14], the present study also revealed higher levels of GLUT1, PKM2, and LDHA in PTC compared with normal tissues.

These dysregulated metabolic enzymes are the basis of cancer cell metabolic reprogramming. Abnormal overexpression of LDHA in several cancers is attributed to the activation of its upstream pathway by cancer-driving mutations [1]. A previous study demonstrated that the HIF $1 \alpha-$ MYC-PGC- $1 \beta$ axis is altered by the $\mathrm{BRAF}^{\mathrm{V} 600 \mathrm{E}}$ mutation, resulting in the inhibition of mitochondrial respiration and the enhancement of aerobic gly- colysis. Moreover, LDHA and PKM2 are elevated in thyroid cancer samples with the $\mathrm{BRAF}^{\mathrm{V} 600 \mathrm{E}}$ mutation [15]. Therefore, the levels of glycolytic enzymes in patients with PTC according to their $\mathrm{BRAF}^{\mathrm{V} 600 \mathrm{E}}$ mutational status, which is the most common mutation found in thyroid cancer, were also evaluated. Contrary to results in previous studies $[15,23]$, only LDHA expression was significantly higher in the $\mathrm{BRAF}^{\mathrm{V} 600 \mathrm{E}}$ mutation group compared with that in the WT BRAF group. It might be due the different regulatory mechanism of each enzyme or sampling criteria [24]. LDHA is considered as a key checkpoint of anaerobic glycolysis. The relationship between the expression of $L D H A$ and the presence of the $\mathrm{BRAF}^{\mathrm{V} 600 \mathrm{E}}$ mutation was also supported by TCGA mRNA data.

To elucidate the role of LDHA in thyroid cancer, further analysis of the relationship between aberrant $L D H A$ expression and the presence of the $\mathrm{BRAF}^{\mathrm{V} 600 \mathrm{E}}$ mutation was performed. LDHA levels were found to be significantly higher in $\mathrm{BRAF}^{\mathrm{V} 600 \mathrm{E}}$ - than in WT BRAF-overexpressing thyroid cells. These cell line pairs are recognized as a suitable model to study the molecular alterations induced by the $\mathrm{BRAF}^{\mathrm{V} 600 \mathrm{E}}$ mutation [25]. To the best of our knowledge, this study is the first to report that the $\mathrm{BRAF}^{\mathrm{V} 600 \mathrm{E}}$ mutant protein causes $L D H A$ overexpression in human thyroid cell lines. We validated this finding using a shBRAF in two human thyroid cell lines, showing that the BRAF ${ }^{\mathrm{V} 600 \mathrm{E}}$ mutation plays a significant role in the regulation of $L D H A$ expression in vitro. Notably, these findings are consistent with a recent report suggesting that $\mathrm{BRAF}^{\mathrm{V} 600 \mathrm{E}}$-mediated metabolic reprogramming increases glycolytic metabolism, which may mediate the Warburg effect [15]. However, we could not reveal the exact mechanism by which the $\mathrm{BRAF}^{\mathrm{V} 600 \mathrm{E}}$ mutation regulated the expression of LDHA in this study. This is a limitation of our study.

It was also demonstrated that high levels of LDHA correlate with aggressive clinical features of PTC, such as extrathyroidal extension, lymph node metastasis, and an advanced cancer stage. These aggressive features are related to the recurrence of PTC, poor response to treatment, and lower overall patient survival [26]. However, our survival analyses showed no differences between groups with different LDHA expression levels. This may be because most thyroid cancers grow slowly and are associated with a very favorable prognosis [21]. Univariate and multivariate analyses revealed that LDHA levels are an independent prognostic marker of lymph node metastasis in PTC. Furthermore, the data suggested that the BRAF ${ }^{\mathrm{V} 600 \mathrm{E}}$ mutation may lead to tumor aggressiveness by altering the expression levels of glycolytic enzymes, such as LDHA. As indicated by recent studies, elevated LDHA levels promote the progression 
of malignant tumors by modulating lactic acid production, glycolysis, reactive oxygen species production, and the expression of numerous cancer-related proteins [1]. To the best of our knowledge, this is also the first study to evaluate and stratify clinicopathological characteristics of thyroid cancer according to the levels of $L D H A$ expression.

$\left[{ }^{18} \mathrm{~F}\right]-\mathrm{FDG}$ positron emission tomography integrated with computed tomography imaging is widely used in the diagnosis and prognostic evaluation of tumors. In particular, the $\left[{ }^{18} \mathrm{~F}\right]-\mathrm{FDG}$ uptake seems to be an indicator of poorly differentiated and highly malignant thyroid cancer [27]. Marked reduction in glucose consumption in LDHA-silenced cells may be accompanied by concurrent decrease in FDG uptake, suggesting a possible association between the FDG uptake and $L D H A$ expression [24, 28]. It was also suggested that the presence of the $\mathrm{BRAF}^{\mathrm{V} 600 \mathrm{E}}$ mutation is associated with the $\left[{ }^{18} \mathrm{~F}\right]-\mathrm{FDG}$ uptake and GLUT1 expression rates in PTC [29]. The relationship between LDHA levels and $\left[{ }^{18} \mathrm{~F}\right]$-FDG uptake was also analyzed but no relevant associations were identified (Supplemental Table S2). Possible reasons for this discordance include (1) $\left[{ }^{18} \mathrm{~F}\right]$-FDG uptake may not necessarily correlate with lactate production, because the upregulation of the pentose phosphate shunt may also result in increased glucose consumption; (2) glucose transporter activity is the major rate-limiting step of $\left[{ }^{18} \mathrm{~F}\right]$-FDG uptake in some tumors; and (3) very high incidence of $\mathrm{BRAF}^{\mathrm{V} 600 \mathrm{E}}$ mutation may have made it difficult to evaluate the relationship [28]. However, further research is still needed.

In summary, this study demonstrated that $L D H A$ overexpression is correlated with aggressive clinical features of PTC. In particular, LDHA could serve as an independent prognostic marker of lymph node metastasis, and $L D H A$ overexpression is associated with the presence of the BRAF ${ }^{\mathrm{V} 600 \mathrm{E}}$ mutation in PTC. At present, the most widely accepted prognostic markers for thyroid cancer are the $\mathrm{BRAF}^{\mathrm{V} 600 \mathrm{E}}$ mutation and mutations in the human telomerase reverse transcriptase promoter [30,31]. However, there is a clear need for novel prognostic markers [7]. The integration of $L D H A$ expression with these well-established markers may help accurately predict poor prognosis in patients with thyroid cancer. To improve our current understanding of thyroid cancer, as well as its management and treatment, more in-depth insights into the role of LDHA are warranted.

\section{CONFLICTS OF INTEREST}

No potential conflict of interest relevant to this article was reported.

\section{ACKNOWLEDGMENTS}

This study was supported by a faculty research grant from Yonsei University College of Medicine (6-2015-0054).

\section{AUTHOR CONTRIBUTIONS}

Conception or design: E.J.B., D.K., K.H.N., K.K. Acquisition, analysis, or interpretation of data: E.J.B., D.K., J.K.K., S.W.K., J.L., J.J.J., K.H.N., W.Y.C. Drafting the work or revising: E.J.B., D.K., K.H.N. Final approval of the manuscript: K.H.N., W.Y.C., K.K.

\section{ORCID}

Eun Jeong Ban https://orcid.org/0000-0001-9153-0506

Daham Kim https://orcid.org/0000-0003-1871-686X

Kee-Hyun Nam https://orcid.org/0000-0002-6852-1190

\section{REFERENCES}

1. Feng Y, Xiong Y, Qiao T, Li X, Jia L, Han Y. Lactate dehydrogenase A: a key player in carcinogenesis and potential target in cancer therapy. Cancer Med 2018;7:6124-36.

2. Woodford MR, Chen VZ, Backe SJ, Bratslavsky G, Mollapour M. Structural and functional regulation of lactate dehydrogenase-A in cancer. Future Med Chem 2020;12:43955.

3. Yao F, Zhao T, Zhong C, Zhu J, Zhao H. LDHA is necessary for the tumorigenicity of esophageal squamous cell carcinoma. Tumour Biol 2013;34:25-31.

4. Girgis H, Masui O, White NM, Scorilas A, Rotondo F, Seivwright A, et al. Lactate dehydrogenase A is a potential prognostic marker in clear cell renal cell carcinoma. Mol Cancer 2014;13:101.

5. Sun X, Sun Z, Zhu Z, Guan H, Zhang J, Zhang Y, et al. Clinicopathological significance and prognostic value of lactate dehydrogenase A expression in gastric cancer patients. PLoS One 2014;9:e91068.

6. Lim H, Devesa SS, Sosa JA, Check D, Kitahara CM. Trends in thyroid cancer incidence and mortality in the United States, 1974-2013. JAMA 2017;317:1338-48.

7. Kim WB, Jeon MJ, Kim WG, Kim TY, Shong YK. Unmet clinical needs in the treatment of patients with thyroid cancer. Endocrinol Metab (Seoul) 2020;35:14-25.

8. Wiltshire JJ, Drake TM, Uttley L, Balasubramanian SP. Sys- 
tematic review of trends in the incidence rates of thyroid cancer. Thyroid 2016;26:1541-52.

9. Chung JH. BRAF and TERT promoter mutations: clinical application in thyroid cancer. Endocr J 2020;67:577-84.

10. Kim TH, Park YJ, Lim JA, Ahn HY, Lee EK, Lee YJ, et al. The association of the BRAF(V600E) mutation with prognostic factors and poor clinical outcome in papillary thyroid cancer: a meta-analysis. Cancer 2012;118:1764-73.

11. Song YS, Lim JA, Park YJ. Mutation profile of well-differentiated thyroid cancer in Asians. Endocrinol Metab (Seoul) 2015;30:252-62.

12. Wen SS, Zhang TT, Xue DX, Wu WL, Wang YL, Wang Y, et al. Metabolic reprogramming and its clinical application in thyroid cancer. Oncol Lett 2019;18:1579-84.

13. Kachel P, Trojanowicz B, Sekulla C, Prenzel H, Dralle H, Hoang-Vu C. Phosphorylation of pyruvate kinase M2 and lactate dehydrogenase $\mathrm{A}$ by fibroblast growth factor receptor 1 in benign and malignant thyroid tissue. BMC Cancer 2015; 15:140.

14. Ciampi R, Vivaldi A, Romei C, Del Guerra A, Salvadori P, Cosci B, et al. Expression analysis of facilitative glucose transporters (GLUTs) in human thyroid carcinoma cell lines and primary tumors. Mol Cell Endocrinol 2008;291:57-62.

15. Gao Y, Yang F, Yang XA, Zhang L, Yu H, Cheng X, et al. Mitochondrial metabolism is inhibited by the HIF1 $\alpha$-MYCPGC-1 $\beta$ axis in BRAF V600E thyroid cancer. FEBS J 2019; 286:1420-36.

16. Cancer Genome Atlas Research Network. Integrated genomic characterization of papillary thyroid carcinoma. Cell 2014;159:676-90.

17. Cancer Genome Atlas Research Network, Weinstein JN, Collisson EA, Mills GB, Shaw KR, Ozenberger BA, et al. The cancer genome atlas pan-cancer analysis project. Nat Genet 2013;45:1113-20.

18. Kim YH, Choi YW, Han JH, Lee J, Soh EY, Park SH, et al. TSH signaling overcomes B-RafV600E-induced senescence in papillary thyroid carcinogenesis through regulation of DUSP6. Neoplasia 2014;16:1107-20.

19. Ko H, Kim S, Jin CH, Lee E, Ham S, Yook JI, et al. Protein kinase casein kinase 2-mediated upregulation of $\mathrm{N}$-cadherin confers anoikis resistance on esophageal carcinoma cells. Mol Cancer Res 2012;10:1032-8.

20. Hanahan D, Weinberg RA. Hallmarks of cancer: the next generation. Cell 2011;144:646-74.

21. Iniguez-Ariza NM, Brito JP. Management of low-risk papil- lary thyroid cancer. Endocrinol Metab (Seoul) 2018;33:18594.

22. Jeon MJ, Kim WG, Kim TH, Kim HK, Kim BH, Yi HS, et al. Disease-specific mortality of differentiated thyroid cancer patients in Korea: a multicenter cohort study. Endocrinol Metab (Seoul) 2017;32:434-41.

23. Grabellus F, Worm K, Schmid KW, Sheu SY. The BRAF V600E mutation in papillary thyroid carcinoma is associated with glucose transporter 1 overexpression. Thyroid 2012;22: 377-82.

24. Miao P, Sheng S, Sun X, Liu J, Huang G. Lactate dehydrogenase A in cancer: a promising target for diagnosis and therapy. IUBMB Life 2013;65:904-10.

25. Kim BA, Jee HG, Yi JW, Kim SJ, Chai YJ, Choi JY, et al. Expression profiling of a human thyroid cell line stably expressing the BRAFV600E mutation. Cancer Genomics Proteomics 2017;14:53-67.

26. Feng C, Gao Y, Wang C, Yu X, Zhang W, Guan H, et al. Aberrant overexpression of pyruvate kinase $\mathrm{M} 2$ is associated with aggressive tumor features and the BRAF mutation in papillary thyroid cancer. J Clin Endocrinol Metab 2013;98: E1524-33.

27. Feine U, Lietzenmayer R, Hanke JP, Held J, Wohrle H, Muller-Schauenburg W. Fluorine-18-FDG and iodine131-iodide uptake in thyroid cancer. J Nucl Med 1996;37: 1468-72.

28. Guo J, Higashi K, Yokota H, Nagao Y, Ueda Y, Kodama Y, et al. In vitro proton magnetic resonance spectroscopic lactate and choline measurements, 18F-FDG uptake, and prognosis in patients with lung adenocarcinoma. J Nucl Med 2004;45:1334-9.

29. Barollo S, Pennelli G, Vianello F, Watutantrige Fernando S, Negro I, Merante Boschin I, et al. BRAF in primary and recurrent papillary thyroid cancers: the relationship with (131) I and 2-[(18)F]fluoro-2-deoxy-D-glucose uptake ability. Eur J Endocrinol 2010;163:659-63.

30. Liu R, Bishop J, Zhu G, Zhang T, Ladenson PW, Xing M. Mortality risk stratification by combining BRAF V600E and TERT promoter mutations in papillary thyroid cancer: genetic duet of BRAF and TERT promoter mutations in thyroid cancer mortality. JAMA Oncol 2017;3:202-8.

31. Xing M. Genetic-guided risk assessment and management of thyroid cancer. Endocrinol Metab Clin North Am 2019; 48:109-24. 